\title{
The Ebola outbreak in the Democratic Republic of the Congo: why there is no 'silver bullet'
}

\author{
Despite new vaccines, therapeutics, and the extensive lessons learned from the West African Ebola outbreak, \\ the DRC's 2018-2020 outbreak has taken nearly 20 months to bring under control. This Comment explores some \\ of the factors that have made this outbreak so complex.
}

\section{Hana Rohan and Gillian McKay}

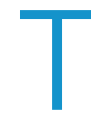
he current outbreak of Ebola in the Democratic Republic of the Congo (DRC) started in August 2018, and there have been a number of extraordinary firsts and successes. But why, despite these, is the outbreak still ongoing after 20 months? Three hundred thousand people have been vaccinated with the recently FDA-licensed Merck EVERBO vaccine and another 20,000 with the Janzen vaccine ${ }^{1}$, despite enormous logistical and regulatory challenges. Investigational therapeutics for confirmed Ebola virus disease (EVD) cases were provided to nearly all confirmed patients either through a compassionate use protocol or as part of a randomized controlled trial, with two of the four therapeutics identified as efficacious ${ }^{2}$ and moved forward to a two-arm trial. Controversial public health interventions that severely disrupted peoples' lives in the West African outbreak, including quarantine, school closures and prevention of mass gatherings, have not been implemented in the North Kivu outbreak, resulting in less severe disruption to social lives and livelihoods. Finally, compared to previous Ebola outbreaks, there has been a significant improvement in the institutionalization of social science data and research during this response, allowing the outbreak response to pay better heed to community feedback and needs.

This EVD outbreak is the tenth occurring in the DRC since the discovery of the virus and is the second largest recorded globally (with 3,453 cases and 2,273 deaths) ${ }^{1}$, eclipsed in size only by the enormous 2013-2016 West African outbreak (28,616 cases and 11,310 deaths) $)^{3}$. There was widespread hope that on April 12th the outbreak would be declared over, however, a new confirmed case identified on April 10 th has reset the countdown clock. The end of the outbreak, when it finally comes, will be a welcome event, particularly now that the DRC (at the time of writing) has already confirmed 180 cases of COVID-19. As social scientists and responders with experience

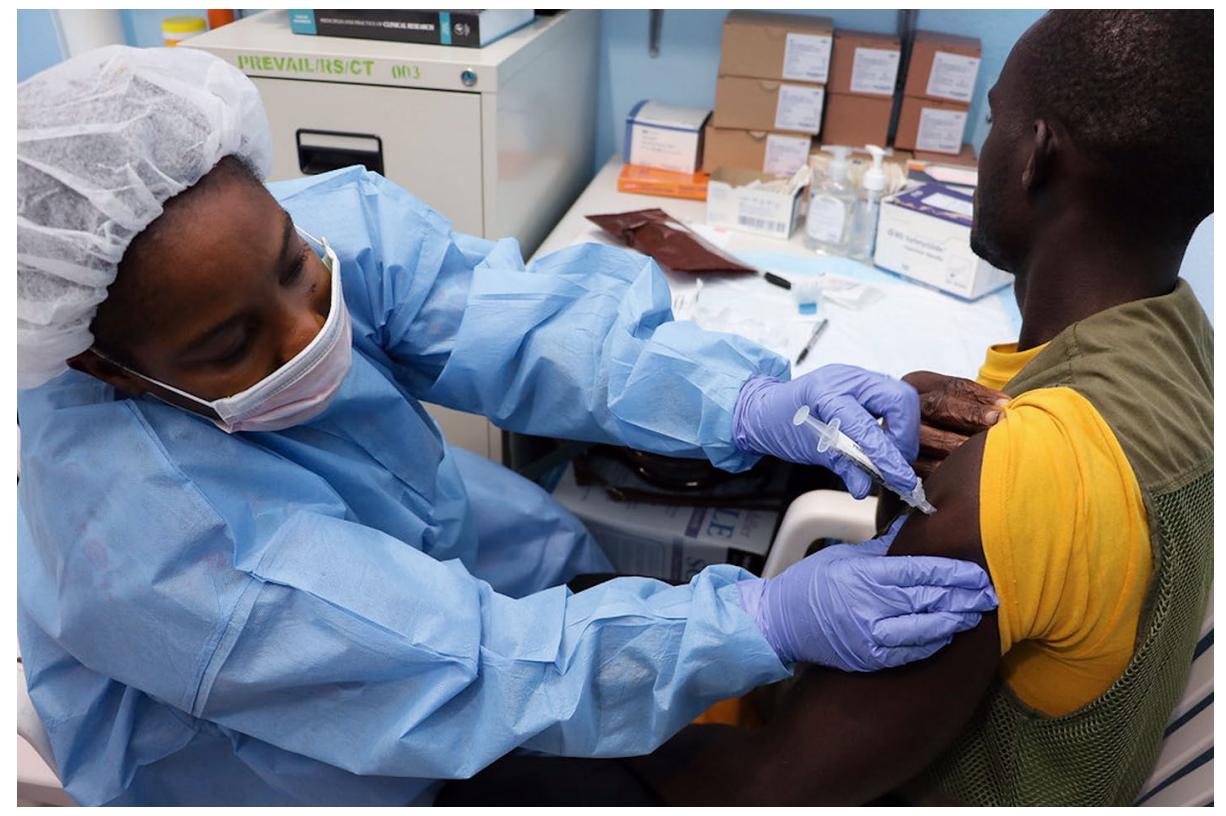

Image: Ebola vaccine study in West Africa. Credit: NIAID, licensed under CC BY 2.0

in both the West African and North Kivu outbreaks, in this Comment we explore the factors that have entrenched the DRC EVD outbreak and taken so many lives, despite new tools, such as vaccines and therapeutics, and the plethora of lessons learned during the West African outbreak.

\section{Health system fragility and fragmentation}

Decades of autocratic rule and armed conflict in the DRC have had a large impact on health system inefficiencies, not least in their effect on overall gross domestic product and, therefore, health spending. Health expenditure per capita is the second lowest in the world, at $\$ 19$ per capita 4 . Health financing is highly dependent on external aid and out-of-pocket payments, and the health system is very fragmented, with many vertical services and programs and limited central-level coordination. The DRC has less than 0.10 doctors for every thousand people ${ }^{5}$, and the health system is therefore significantly understaffed, with more qualified staff in urban areas ${ }^{6}$.

The gaps that arose in the public health sector because of political and civil instability were partially filled by a growing private - often faith-based - health care industry that remains an important provider within the DRC's health ecosystem. Available data indicate that the private sector accounted for $46 \%$ of the DRC's 469 hospital structures in 2017. Faith-based organizations are estimated to manage or comanage (with the Ministry of Health) approximately $40 \%$ of health zones and $50 \%$ of all facilities in the country ${ }^{7}$.

The private health care sector faces many of the same challenges as the public health care system, particularly with regards to 
the availability of critical commodities, a shortage of trained staff and the (in)ability of patients to pay for care ${ }^{7}$. Health system fragmentation contributes to existing weaknesses in health information and management systems that are exacerbated by poor infrastructure ${ }^{8}$. Weak and fragmented health information systems mean that routine reporting cannot be relied on in the event of an outbreak like EVD, placing all the surveillance onus onto the outbreak response itself. Fragmentation also affects other outbreak response activities, such as infection prevention and control (IPC); it is extremely difficult to appropriately quantify and supply personal protective equipment (PPE) and other IPC materials like hand-washing buckets if there is no clear information on the number or location of health facilities.

Prior to the outbreak, late presentation at health facilities was common, with access problems and fees acting as barriers ${ }^{9}$. EVD response initiatives designed to encourage early care seeking (such as the introduction of free health care in some clinics) were hampered by many of the health system challenges described above. In particular, there were issues around the supply of medical commodities as well as insufficient numbers of health workers ${ }^{10}$ to cope with the increased demand that inevitably arose from free care being made available ${ }^{11}$, potentially exacerbating distrust in local facilities. There is widespread fear and distrust of specialized EVD infrastructure (including isolation and treatment facilities), and there have been reports of reduced trust in non-EVD facilities, for fear of being misdiagnosed with EVD or of nosocomial exposure to the disease $\mathrm{e}^{12}$. Communities have continued to approach care seeking pragmatically, often self-medicating or consulting with pharmacies or traditional healers to avoid traveling long distances or in an attempt to reduce costs. For communities affected by ongoing conflict, access to health care has been a major challenge since long before the current outbreak, with many health facilities located in insecure areas or closed altogether ${ }^{9}$. This insecurity has continued into the outbreak: it is well documented that there have been attacks on Ebola treatment centers and frontline workers, inevitably affecting access to EVD care as well as contact tracing and other surveillance activities, which in turn affects the impact of response control interventions ${ }^{13}$.

\section{Disenfranchisement, mistrust and neglect}

The current outbreak of EVD in North Kivu and Ituri provinces, in the eastern region of DRC, has taken place against the backdrop of a war that has lasted more than twenty years - one of the continent's longest running conflicts ${ }^{14}$. North Kivu's long war has contributed to health system fragility but also to entrenched mistrust in local authorities, the national government and international actors that have had implications for the EVD response. A survey conducted in the towns of Beni and Butembo in September 2018 showed that only around $30 \%$ of respondents believed that local authorities represented their interests and that around 25\% of respondents believed that EVD is not real. These factors were shown to be associated with a reduced likelihood of seeking formal health care and adopting preventive behaviors ${ }^{15}$.

The region is a mainstay of opposition to the national government and home to multiple armed groups that have been prone to increasing bouts of violence since 2013, killing nearly 1,000 people and displacing more than 180,000. The Congolese state and the UN peacekeeping mission (MONUSCO) are perceived to have failed to protect local citizens from this violence, and, therefore, neither the government nor international actors are trusted ${ }^{16}$. On top of violence, the North Kivu and Ituri provinces also suffer from entrenched poverty, food insecurity and a large internally displaced population. A severe measles outbreak in the province has claimed twice as many lives as EVD, and cholera is a recurring problem ${ }^{17}$. A perception that the response is uninterested in improving health outcomes beyond EVD has been exacerbated by the EVD response's recruitment of health workers and their attendant diversion from routine delivery of health services and by the imbalance in levels of international attention and funding for $\mathrm{EVD}^{18}$. In this context, the highly visible and expensive EVD response is seen by many residents as further evidence that the region has been neglected. Events such as the cancellation of the presidential and legislative elections in the EVD-affected region ramped up mistrust further, being interpreted by many as intended to rig the vote rather than to control the outbreak $^{19}$. In turn, it is unsurprising that EVD is considered by many to have been deliberately introduced to the region for political reasons or to not exist at all. This skepticism therefore extends to the EVD response itself, with consequences for its operational efficacy, and has been further compounded by the perception that the response is predominantly led by outsiders and that it failed to properly engage with local communities ${ }^{20}$.

Insecurity and a climate of mistrust, on top of poor road, telecommunications and health system infrastructure, made responding to the outbreak extremely challenging and unsafe. Road travel to some areas is considered so dangerous that armored vehicles are required, with health staff wearing flak jackets and helmets. Poor mobile network access occasionally delayed reports of new cases, resulting in sick individuals waiting long periods to be collected and taken to facilities for treatment. Prior to this outbreak, few health facilities had adequate infection prevention and control infrastructure, with limited or non-existent trained staff, PPE, running water and waste disposal.

\section{Mutually reinforcing crises}

The ongoing insecurity has also had a direct impact on driving the outbreak forward. There are likely more than 120 armed groups operating in North and South Kivu. Some of the most violent groups are located in the 'Grand Nord' of North Kivu, which includes the town of Beni, where much of the current outbreak has been concentrated. Civilian massacres in this area drive constant population movement in and out of the town, leading to repeated new outbreaks in the same area, even after they have been declared Ebola-free.

The conflict has also led to the establishment of indigenous self-defense groups, often referred to collectively as "Mai-Mai" (although not all armed groups identify as Mai-Mai, and, in reality, there is substantial variation in the actors, funding, allegiance and level of organization of these groups). One of the roles of the Mai-Mai is to protect the local population against attacks by external armed groups. However, mistrust of the Ebola response has sometimes led the Mai-Mai to view the response as just such an external threat and to attack the response itself, including Ebola treatment centers and health care workers. When there are significant attacks on EVD response infrastructure, the response inevitably has to reduce activity for several days to wait for the insecurity to decline and for additional security measures to be instituted. These pauses or slowdowns in response activity have been shown to exacerbate the number of Ebola cases, as preventive activities like IPC, surveillance and community engagement are interrupted, facilitating the spread of the virus ${ }^{21}$.

Just as the conflict has had direct effects on driving up the outbreak, the outbreak and its response have also exacerbated insecurity. UN response staff, fearing attacks, have at times been required to operate with the accompaniment of security forces (local police, the national army and MONUSCO). This perceived close 


\section{Box 1| Accessing care during mutually reinforcing crises}

Pregnant women in the DRC particularly those in more remote areas - will sometimes await labor and delivery at 'maternity waiting homes', where they can stay and rest while waiting for labor to begin, to ensure that they will have access to trained health workers to support them through delivery. Most women will go to these maternity waiting homes two weeks prior to their due date. The outbreak, layered on top of the conflict, has disrupted these routine health care decisions. One woman at a maternity waiting home (located at a hospital in a high-transmission area) was from a village with a nearby clinic that had, due to the outbreak, experienced attacks on health care workers who were thought to be 'collaborating' with the EVD response. Her clinic was therefore closed, and the health workers had fled, leaving her without a safe location to deliver close to her home. Fearing that the route to the maternity waiting home would become impassable due to rebel activity, and leaving behind her children and husband, she had arrived at the maternity waiting home a whole month prior to her due date, increasing her risk of exposure to EVD in that setting. This anecdote illustrates the ways in which these mutually reinforcing crises, set against the backdrop of a fragile health system, can have very real implications for health decision making and potentially for health outcomes. relationship between security forces and health actors has facilitated the legitimacy of attacks on EVD response staff. The huge influx of 'Ebola money' (funds spent locally on the response), which is unequally dispersed to elites, local businesses and workers, may be inadvertently funding armed groups, given the fluid nature of their membership.

At times, the two crises have become entirely mutually reinforcing, with consequences for health care access (see Box 1). In Beni in November and December 2019, there were significant armed group attacks on local civilian populations by the Allied Democratic Forces (ADF), a violent armed group, in retaliation for the Congolese army's military offenses against them $^{22}$. More than 200 civilians were killed, and, in response, civilians in Beni attacked the MONUSCO base there, arguing that the presence of the UN forces was not sufficiently protecting the local population. One UN base was destroyed, and while this attack does not appear to have been directly targeted at the EVD response, MONUSCO's provision of security services for the response has complicated the perception of relationships between different institutional, military and EVD response actors. The attack on the UN base forced the evacuation of many EVD response actors and a near halt of response activities for several days. Prior to this, case numbers in Beni and surrounding areas had been declining, and many had hoped that, in that area at least, the fight against EVD was nearing an end. The attack and consequent disruption to response activities led to a resurgence in cases and likely set the response back several months ${ }^{23}$.

\section{Evidentiary inertia}

Notwithstanding this extremely fraught operating environment for the Ebola response, its size and complexity has meant that it is not always primed to adapt quickly to new evidence or research. There are examples of flexibility from this outbreak: the safe and dignified burials program has implemented community-based burial teams in highly insecure areas who are trained and supplied by the response but who respond locally and as a community when a death occurs, limiting community interactions with 'outsiders' that could endanger responders or, indeed, community members themselves, by making them targets for militia groups. However, large humanitarian responses can struggle to rapidly take up and implement new evidence and research findings. A rapid operational research project in Butembo, a city in North Kivu, identified that community members were extremely unhappy about infection prevention and control activities that entailed the burning of household belongings outside the homes of families with confirmed Ebola cases; this response activity was considered deeply intrusive as well as stigmatizing. Despite these research findings, it took many months for a change in IPC protocols regarding the disposal of potentially infectious items. Similarly, during the West African EVD outbreak, the use of spray chlorine was shown to be not only ineffective as an IPC measure but also to have negative health outcomes for those in the vicinity of spraying ${ }^{24}$. Spraying chlorine as a disinfection measure continued to be widely used in the North Kivu outbreak until about a year into the response, when the protocol was changed. Pregnant and lactating women were initially excluded from the Merck EVD vaccine compassionate use protocol used in North Kivu out of concerns that women could spontaneously abort or that infants could be harmed. Some of the excluded women were high-risk contacts but were not permitted to receive the vaccine, despite operational research showing that they understood the risks and would prefer to be given the choice about whether to take the vaccine.

This 'evidentiary inertia' emanates from reasonable concerns about the logistical challenges of changing response-wide protocols as well as the belief that redesigning standard operating procedures (SOPs) and retraining staff would simply be too great a challenge, despite part of their purpose being to ensure that outbreak responses are evidence led.

It is difficult to know how much bigger this outbreak would have been in the absence of the ERBEVO vaccine ${ }^{25}$, and a significant number of lives have undeniably been saved through the use of new therapeutics. These technologies are game changers in the fight against Ebola and will, without a shadow of a doubt, improve outbreak responses and individual health outcomes in future outbreaks. However, in this outbreak, their first major rollout, the challenge has gone well beyond the 'normal' complexity of responding to a disease that disrupts economies, social and familial ties and health systems and, therefore, well beyond the mandate of the World Health Organization. Response organizations and individuals involved in the North Kivu outbreak should be lauded for their bravery and tenacity. However, the humanitarian community has long wondered what would happen when a major viral hemorrhagic fever outbreak occurred in an active conflict zone, and North Kivu has shown that, collectively, we still have much to learn about responding within such entrenched complexity.

COVID-19 transmission, severity and mortality rates are all likely to play out differently in countries like the DRC, partly as a result of that complexity but also because of the stark reality of extremely limited critical care bed capacity. One-size-fits-all approaches to controlling the disease are therefore unlikely to work in many different settings. The DRC is making strides in reorienting its health emergency response away from Ebola and toward the new threat of severe respiratory disease, but unless lessons from EVD and beyond are considered and applied, COVID-19 is likely to highlight, once again, global inequities in access to health 
and the outrageous cost of it for those in low-and-middle-income countries. As the world faces important decisions about how to control and mitigate the COVID-19 pandemic, particularly in vulnerable settings, it is critical that we learn from the North Kivu EVD experience by being more adaptable, embedding community listening into response decision-making processes, and really investing in fragile health systems for reasons of equity, stability and global health security.

\section{Hana Rohan (D) $1 \otimes$ and Gillian McKay (D) 2 \\ ${ }^{1}$ UK Public Health Rapid Support Team, London School of Hygiene and Tropical Medicine, London,} UK. ${ }^{2}$ Department of Global Health and Development, London School of Hygiene and Tropical Medicine, London, UK

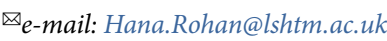

Published online: 21 April 2020

https://doi.org/10.1038/s41590-020-0675-8

References

1. Ebola Virus Disease Democratic Republic of the Congo: External Situation Report 85 (World Health Organization, 2020).

2. Mulangu, S. et al. N. Engl. J. Med. 381, 2293-2303 (2019).

3. Ebola Virus Disease Situation Report - 10 June 2016 (World Health Organization, 2016).

4. Global Health Expenditure Database (World Health Organization, accessed 11 December 2019); http://apps.who.int/nha/database/ Select/Indicators/en)/en

5. The Central Intelligence Agency. CIA World Factbook: Democratic Republic of Congo (13 March 2020); https://www.cia.gov/library/ publications/the-world-factbook/geos/print_cg.html
6. Ntembwa, H. K. \& Van Lerberghe, W. Improving Health System Efficiency: Democratic Republic of the Congo: Improving Aid Coordination in the Health Sector (World Health Organization, 2015).

7. Brunner, B. et al. The Role of the Private Sector in Improving the Performance of the Health System in the Democratic Republic of Congo (The World Bank, 2018).

8. Gavi. A plan of action: boosting immunisation coverage in DRC. Gavi The Vaccine Alliance https://www.gavi.org/vaccineswork/ country-stories/plan-action-boosting-immunisation-coverage-drc (2020)

9. Bedford, J. \& Social Science in Humanitarian Action. Key Considerations: The Context of North Kivu Province, DRC (UNICEF, IDS \& Anthrologica, 2018).

10. Bardosh, K., Gercama, I. \& Bedford, J. Social Science and Behavioural Data Compilation, DRC Ebola Outbreak, November 2018-February 2019 (UNICEF, IDS \& Anthrologica, 2019).

11. Policy Options for Temporary Fee Elimination Programs for Ebola Response in DRC: Learning from International Experience and from Recent Efforts in DRC - Inception Report (World Bank, 2019).

12. McKay, G. et al. Not All That Bleeds Is Ebola: How Has the DRC Ebola Outbreak Impacted Sexual and Reproductive Health in North-Kivu? (The International Rescue Committee, 2019).

13. Tariq, A., Roosa, K., Mizumoto, K. \& Chowell, G. Epidemics 26,128-133 (2019).

14. DR Congo Country Profile. BBC (10 January 2019); https://www. bbc.co.uk/news/world-africa-13283212

15. Vinck, P., Pham, P. N., Bindu, K. K., Bedford, J. \& Nilles, E. J. Lancet Infect. Dis. 19, 529-536 (2019).

16. Vogel, C. et al. Cliches Can Kill in Congo. Foreign Policy (30 April 2019); https://foreignpolicy.com/2019/04/30/ cliches-can-kill-in-congo-grand-nord-north-kivutropes-conflict-ebola-response/

17. Potter, C. Beyond Ebola: DRC Battles Measles and Cholera. Outbreak Observatory https://www. outbreakobservatory.org/outbreakthursday-1/7/18/2019/ beyond-ebola-drc-battles-measles-and-cholera (2019).

18. Arie, S. Br. Med. J. 366, 14522 (2019).

19. Trapido, J. Lancet Infect. Dis. 19, 457-458 (2019).

20. Sweet, R. \& Bedford, J. WhatsApp and local media (Grand Nord) -9-18 September 2018: Reluctance, Refusal, Resistance and the Politicisation of the Ebola Response. Social Science in Humanitarian Action Platform (UNICEF, IDS \& Anthrologica, 2018).
21. Wannier, S. R. et al. Epidemics 28, 100353 (2019).

22. Atrocity Alert No. 182: Democratic Republic of the Congo, Chile and the ICC Assembly of States Parties (Global Centre for the Responsibility to Protect, 2019); https://reliefweb. int/report/democratic-republic-congo/atrocity-alert-no182-democratic-republic-congo-chile-and-icc

23. Ebola Virus Disease: Democratic Republic of the Congo External Situation Report 69 (World Health Organization, 2019)

24. Mehtar, S., Bulabula, A. N. H., Nyandemoh, H. \& Jambawai, S. Antimicrob. Resist. Infect. Control 5, 45 (2016).

25. Preliminary Results on the Efficacy of $r V S V-Z E B O V-G P$ Ebola Vaccine Using the Ring Vaccination Strategy in the Control of an Ebola Outbreak in the Democratic Republic of the Congo: An Example of Integration of Research into Epidemic Response (World Health Organization, 2019); https://www.who.int/csr/resources/publications/ebola/ vaccines/en/

Acknowledgements

The UK Public Health Rapid Support Team is funded by aid from the UK Department of Health and Social Care and is jointly run by Public Health England (PHE) and the London School of Hygiene \& Tropical Medicine (LSHTM). The University of Oxford and King's College London are academic partners. The views expressed in this publication are those of the authors and not necessarily those of the National Health Service, the National Institute for Health Research or the Department of Health and Social Care. Gillian McKay is supported by the Pierre Elliott Trudeau Foundation; she conducted research on the DRC outbreak as part of a project funded by Elhra's Research for Health in Humanitarian Crises (R2HC) programme, which is funded by the UK Department for International Development (DFID), Wellcome, and the UK National Institute for Health Research (NIHR).

\section{Competing interests}

The authors declare no competing interests. 